\title{
The Topology of Hypersurfaces Moving by Mean Curvature
}

\author{
BRIAN WHITE
}

\section{Introduction.}

Let $M$ be a smooth compact embedded hypersurface in a riemannian manifold $N$. Then there is a $T>0$ and a smooth map

$$
f:[0, T) \times M \rightarrow N
$$

such that $f(0, \cdot)$ is the identity map, $M_{t}=f(t, M)$ is smoothly embedded for each $t$, and

$$
\frac{d}{d t} f(t, x) \equiv H(t, x)
$$

where $H(t, x)$ is the mean curvature of $M_{t}$ at $f(t, x)$. Examples show that the $M_{t}$ may become singular as $t \rightarrow T$, so that the equation (1) ceases to make sense classically. Nevertheless, there are various notions of weak solution that allow one to continue the flow $\left\{M_{t}\right\}$ beyond $t=T$. Of course for $t<T$, the surfaces $M_{t}$ are all topologically equivalent (indeed isotopic). However, examples show that the topological type of $M_{t}$ can change after singularities. This paper addresses the question: what topological changes are possible?

Associated with any one parameter family of surfaces $M_{t}(0 \leq t \leq T)$ in the ambient space $N$, we have a closed subset

$$
\mathcal{M}=\left\{(t, x): x \in M_{t}\right\}
$$

of spacetime $\mathbf{R}^{+} \times N$. Conversely, any closed set $\mathcal{M}$ in spacetime can be regarded as a 1-parameter family $M_{t}$ of sets moving in space:

$$
M_{t}=\{x:(t, x) \in \mathcal{M}\}
$$

In this paper, we say that the set $\mathcal{M}$ is a weak mean curvature flow (or weak flow, for short) if it does not violate the maximum principle. Here " $\mathcal{M}$ violates the maximum principle" means there exists a smooth classical flow

$$
\mathcal{M}^{\prime}=\left\{(t, f(t, x)): x \in M^{\prime}, a \leq t \leq b\right\}
$$


(with $f$ satisfying (1)) such that $\mathcal{M}$ and $\mathcal{M}^{\prime}$ are disjoint initially (at $t==a$ ) but intersect later. Such weak solutions are also called subsolutions to mean curvature flow in [I1, §10.1], [I3]. Classical solutions to the mean curvature flow are weak flows in this sense, as is any Brakke flow of integral varifolds. That is, if the $M_{t}$ are the supports of any family of integral varifolds flowing by mean curvature according to Brakke's definition (see [B] or $[\mathrm{I} 1, \S 6]$ ), then the associated $\mathcal{M}$ is a weak solution (see [I1, §10.8]).

In general, there may be more than one weak solution generated by a given initial set $M_{0}$. However, there is one weak solution $\mathcal{M}$ generated by $M_{0}$ that contains all other weak solutions generated by $M_{0}$. This weak solution is called the biggest flow or the level set flow generated by $M_{0}$.

Our main results (theorems 5.2, 5.4, 6.1,6.2) can be summarized as follows.

Theorem 1. Let $\mathcal{M}$ be a closed subset of spacetime $\mathbf{R}^{+} \times N$ and let $W$ be its complement. Let

$$
W[\tau]=\{(t, x) \in W: t=\tau\}
$$

and

$$
W[0, T]=\{(t, x) \in W: 0 \leq t \leq T\}
$$

(i). If $\mathcal{M}$ is any level set flow, then the inclusion $\iota_{0}: W[0] \rightarrow W[0, T]$ induces an isomorphism on $\mathbf{H}_{0}$ :

$$
\left(\iota_{0}\right)_{\#}: \mathbf{H}_{0}(W[0]) \stackrel{\cong}{\rightrightarrows} \mathbf{H}_{0}(W[0, T])
$$

(ii). If $\mathcal{M}$ is any level set flow, then every loop in $W[0, T]$ is homotopic to a loop in $W[0]$.

(iii). Suppose $N$ is a complete $(n+1)$-dimensional riemannian manifold with ricci curvature bounded below. If $\mathcal{M}$ is any weak flow generated by a set $M_{0}$ at time 0 , then the inclusion $\iota_{T}: W[T] \rightarrow W[0, T]$ induces monomorphisms on $\mathbf{H}_{n-1}$ and on $\mathbf{H}_{n}$ :

$$
\begin{aligned}
\left(\iota_{T}\right)_{\#}: \mathbf{H}_{n-1}(W[T]) & \mapsto \mathbf{H}_{n-1}(W[0, T]) \\
\left(\iota_{T}\right)_{\#}: \mathbf{H}_{n}(W[T]) & \mapsto \mathbf{H}_{n}(W[0, T])
\end{aligned}
$$

Heuristically, (i) says that two distinct connected components of the complement of $M_{0}$ can not become connected together later (though a connected component can become disconnected; see \$2.4), and that connected 
components cannot appear out of nowhere. Conclusion (ii) says that loops in the complement of $M_{t}$ cannot appear out of nowhere. Conclusion (iii) implies (among other things) that an (n-1)-cycle in the complement of $M_{t}$ cannot suddenly cease to bound an $n$-chain in the complement, and similarly for a $n$-cycle.

The conclusions are perhaps easiest to understand for two-dimensional surfaces moving in a 3-manifold. For example, if we let $\mathcal{M}$ be the level set flow in $\mathbf{R}^{3}$ generated by some set $M_{0}$ at time 0 , then (ii) and (iii) imply that $\operatorname{dim} \mathbf{H}_{1}(W[t])$ is a monotonically non-increasing function of $t$. Consequently if $M_{t(1)}$ and $M_{t(2)}$ are both smoothly embedded and if $t(1)<t(2)$, then the genus of $M_{t(2)}$ is less than or equal to the genus of $M_{t(1)}$. (If $M_{0}$ is mean-convex, then $M_{t}$ is known to be smooth and embedded for almost all $t[\mathrm{~W} 1]$.

One can also consider mean curvature evolution of surfaces with boundary. The boundary may either be held stationary or moved in a prescribed way. Conclusions (i) and (ii) remain true in this setting.

Level set flow in euclidean space was introduced for numerical analysis by Osher and Sethian [OS]. The mathematical theory was developed by Chen, Giga, and Goto [CGG] and by Evans and Spruck [ES]. Later it was developed from a different point of view by Soner [S] and extended to general ambient manifolds by Ilmanen [I2] (See also [I1, §10] and [I3].) Since those papers do not consider surfaces with boundary, we give a brief self-contained development of the theory of weak and level set flows that includes moving surfaces with boundaries.

The results of this paper also hold for some other geometrical evolution equations; see $\S 8$. There are also some analogous results for minimal surfaces [W2].

\section{Examples.}

In this section we present some examples of topological changes. A smooth compact hypersurface bounding a region in $\mathbf{R}^{n+1}$ is called meanconvex if at each point its mean-curvature vector is a non-negative multiple of the inward-pointing unit normal. If the initial surface $M_{0}$ of a level set flow is mean-convex, then for each $0 \leq \tau<t, M_{t}$ is contained in the region bounded by $M_{\tau}$. In this case the various definitions of mean curvature flow essentially coincide, except that in a Brakke flow or in a weak flow (subsolution), connected components are allowed to vanish suddenly at any time. 


\section{Example 1: shrinking sphere.}

An initial round sphere shrinks (under the level set flow) through concentric spheres to a point in a finite time, and then disappears. The set $\mathcal{M}$ in spacetime is an upside down paraboloid. This is of course consistent with theorem 1.

Note however that we can let the sphere vanish at some time $\tau<T$ and still have a Brakke flow (and therefore a weak flow):

$$
\mathcal{M}^{\prime}=\{(t, x) \in \mathcal{M}: t \leq \tau\}
$$

In this example, two disconnected components of the complement of $M_{0}$ join up later on. Thus for general Brakke flows the map $\left(\iota_{0}\right)_{\#}$ on $\mathbf{H}_{0}$ in theorem 1 need not be injective.

\section{Example 2: fattening.}

Let $M_{0}$ be a smooth figure 8 curve in the plane, and let $\mathcal{M}$ be the le:vel set flow it generates. It is well-known that $\mathcal{M}$ has a nonempty interior. Now the topological boundary $\mathcal{M}^{\prime}$ of $\mathcal{M}$ is also a weak solution generated by $M_{0}$. Points in the interior of $\mathcal{M}$ lie in the complement of $\mathcal{M}^{\prime}$, but cannot be connected (in the complement of $\mathcal{M}^{\prime}$ ) to points at time 0 . Thus for general weak solutions (and similarly for Brakke flows) the homomorphism of $\mathbf{H}_{0}$ in theorem 1 (i) need not be surjective.

\section{Example 3: loops appearing.}

Let $\mathbf{T}$ be the torus in $\mathbf{R}^{3}$ formed by revolving around the $y$-axis a disk in the $x y$ plane of radius $\epsilon$ centered at $(1,0)$. Let $\mathbf{D}$ be a disk of radius $\epsilon$ with boundary in $\mathbf{T}$. Let $M_{0}$ be the union of $\mathbf{T}$ and $\mathbf{D}$. Let $M_{t}$ flow in the natural way as a varifold for a short time $\tau$. Let the disk vanish at time $\tau$, and then continue the flow in the natural way. For $t$ slightly greater than $\tau$, the complement of $M_{t}$ will contain a loop that does not arise from any loop in the complement of $M_{0}$. This shows that conclusion (ii) of theorem 1 does not hold for Brakke flows (or for weak flows).

\section{Example 4: dumbell.}

Let $n \geq 2$, and consider the surface $M_{0}$ formed by joining two $n$-spheres in $\mathbf{R}^{n+1}$ by a thin rotationally symmetric tube. It is easy to make $M_{0}$ mean 
convex. If the tube is thin enough, then under the level set flow it will pinch off in a finite time, separating the surface into two or more components, each of which is topologically a sphere. Note the behavior is consistent with theorem 1. (See [AAG] for a detailed analysis of this example.)

\section{Example 5: generalized dumbell.}

Let $J$ be union of a pair of unit disks on the x-axis and a long thin rectangle joining their centers:

$$
J=\mathbf{B}^{2}((a, 0), 1) \cup \mathbf{B}^{2}((-a, 0), 1) \cup([-a, a] \times[-\epsilon, \epsilon])
$$

Here $a$ should be large.

Fix integers $p \geq 1$ and $q \geq 2$. Let us represent points in $\mathbf{R}^{p+q}=\mathbf{R}^{p} \times \mathbf{R}^{q}$ as $(x, y)$ where $x=\left(x_{1}, \ldots, x_{p}\right)$ and $y=\left(y_{1}, \ldots, y_{q}\right)$. Let $\tilde{J}$ be the result of rotating $J$ (thought of as lying in the $x_{1} y_{1}$ plane) about the $x=0$ and $y=0$ planes. Symbolically:

$$
\tilde{J}=\left\{(x, y) \in \mathbf{R}^{p+q}:(|x|,|y|) \in J\right\}
$$

Let $M_{0}$ be the least area hypersurface containing $\tilde{J}$. Then $M_{0}$ will be a mean convex $C^{1,1}$ surface. If $a$ is large, then $M_{0}$ will coincide with the boundary of $\tilde{J}$ in a large ball around the origin.

Let $M_{t}$ be the result of letting $M_{0}$ flow for time $t$ under the level set flow. If $a$ is sufficiently large, it is not hard to show that at a certain finite time $T, M_{T}$ will no longer touch the $x=0$ plane, but will not yet have vanished. (One uses Huisken's monotonicity formula $[\mathrm{H}]$ or Brakke's clearing out lemma $[\mathrm{B}, 6.3]$ to show that it does not touch the $x=0$ plane, and spherical barriers to show that it has not yet disappeared.) Indeed, we can arrange for the region enclosed by $M_{T}$ to contain the $(p-1)$-sphere

$$
\mathbf{S}=\partial \mathbf{B}^{p}(0, a) \times[0]^{q}
$$

This sphere does not bound any $p$ chain in the interior of $M_{T}$ (since any such $p$ chain must cross the $x=0$ plane). On the other hand, $\mathbf{S}$ (which belongs to the interior of all the preceding $M_{t}$ 's) does bound a chain in the interior of $M_{0}$. This shows that conclusion (iii) of theorem 1 does not hold for any homology groups $\mathbf{H}_{k}$ with $k<n-1$.

Similarly, let $\mathbf{S}^{\prime}$ be the union of $[0]^{p} \times \mathbf{B}^{q}(0, a+2)$ with a $q$-dimensional hemisphere having the same boundary. Then $\mathbf{S}^{\prime}$ is a $q$-cycle in the complement of $M_{T}$, and $\mathbf{S}^{\prime}$ is not the descendent of any $q$-cycle in the complement of 
$M_{0}$. This shows that conclusion (ii) of theorem 1 does not hold for $k$-cycles with $k>1$.

\section{Classical Flows.}

Definition. Let $M$ be a compact $n$-manifold with or without boundary and let $\operatorname{int}(M)=M \backslash \partial M$ be its interior. Let $N$ be a riemannian $(n+1)$ manifold, and let

$$
f:[a, b] \times M \rightarrow N
$$

be a continuous one-one map that is smooth on $(a, b] \times \operatorname{int} M$ such that $f(t, \cdot)$ smoothly embeds $\operatorname{int}(M)$ for each $t \in(a, b]$. Suppose that for each $(t, x) \in(a, b] \times \operatorname{int}(M)$,

$$
\left(\frac{d}{d t} f(t, x)\right)^{\perp}=H(t, x)
$$

where ${ }^{\perp}$ denotes the component normal to $f(t, M)$ and $H(t, x)$ denotes the mean curvature of $f(t, M)$ at $(t, x)$. Then for any $c \in(a, b)$, we say that the set

$$
\mathcal{M}:=\{(t, f(t, x)): t \in[a, b], x \in M\}
$$

is a classical mean curvature flow (or classical flow, for short).

The heat boundary $\partial \mathcal{M}$ of $\mathcal{M}$ is the image under $(t, x) \mapsto(t, f(t, x))$ of

$$
([a] \times M) \cup([a, b] \times \partial M)
$$

Let $\mathcal{M}$ be a classical flow and let $X=(\tau, p)$ be in $\mathcal{M} \backslash \partial \mathcal{M}$. Let $M_{t}$ be $\{x:(t, x) \in \mathcal{M}\}$. Choose a coordinate system for $N$ in which $p$ is at the origin and in which the tangent plane to $M_{\tau}$ at $p$ is not vertical (i.e., does not contain the standard basis vector $(0,0, \ldots, 0,1)$.) Then there will be a cylinder $\overline{\mathbf{B}}^{n}(0, r) \times(-h, h)$ such that for $t=\tau$, the intersection of $M_{t}$ with the cylinder is the graph of a smooth function $u_{t}: \overline{\mathbf{B}}^{n}(0, r) \rightarrow \mathbf{R}$. It follows that this also holds for all $t$ sufficiently near $\tau$. Of course $(t, x) \rightarrow u_{t}(x)$ is a solution of the non-parametric mean curvature flow equation (which is a quasilinear parabolic equation).

Lemma 3.1 (Maximum Principle Lemma). Let $\mathcal{M}_{i}(i=1,2)$ be two classical flows. If they intersect, then they intersect at a point that belongs to one of the two heat boundaries.

Indeed, if $X \in \mathcal{M}_{1} \cap \mathcal{M}_{2}$, then there is a connected subset $K$ of $\mathcal{M}_{1} \cap \mathcal{M}_{2}$ such that $K$ contains $X$ and some point in $\partial \mathcal{M}_{1} \cup \partial \mathcal{M}_{2}$. 
Proof. Let $\mathcal{S}$ be the intersection of the parabolic interiors of the $\mathcal{M}_{i}$. We claim that if $X=(\tau, x) \in \mathcal{S}$, then there exist points $X^{\prime} \in \mathcal{S}$ arbitrarily close to $X$ and strictly preceding $X$. For suppose not. Then we can choose $\epsilon>0$ and a neighborhood $U \subset N$ of $x$ such that the set

$$
(t-\epsilon, t) \times U
$$

contains no parabolic boundary points of the $\mathcal{M}_{i}$ and no points of $\mathcal{S}$. Hence $\mathcal{M}_{1}$ and $\mathcal{M}_{2}$ do not intersect in the set $\left(^{*}\right)$. But now the classical strong maximum principle (applied to the functions $u_{t}$ corresponding to $\mathcal{M}_{1}$ and $\mathcal{M}_{2}$ ) implies that they do not intersect at $X$ either, a contradiction. This proves the claim. Now the result is a consequence of the following simple point-set topology lemma. (Let $B$ be $\mathcal{M}_{1} \cap \mathcal{M}_{2}$ and let $A$ be the intersection of $B$ with $\partial \mathcal{M}_{1} \cup \partial \mathcal{M}_{2}$.)

Lemma 3.2. Let $A$ and $B$ be compact subsets of $\mathbf{R} \times N$ such that $A \subset B$. Suppose for every $X \in B \backslash A$, there exists points $Y \in B$ that are arbitrarily close to $X$ and prior to it. Then for every $X \in B$, there exists a closed connected subset $K \subset B$ such that $K$ contains $X$ and a point $Z \in A$.

Proof. For $\epsilon>0$, let $\mathcal{F}_{\epsilon}$ be the family of subsets $S \subset B$ such that

(i). $X \in S$, and

(ii). If $S=S^{\prime} \cup S^{\prime \prime}$ with $S^{\prime}$ and $S^{\prime \prime}$ nonempty, then there exist points $Y^{\prime} \in S^{\prime}$ and $Y^{\prime \prime} \in S^{\prime \prime}$ with $\operatorname{dist}\left(Y^{\prime}, Y^{\prime \prime}\right) \leq \epsilon$.

By the Hausdorff maximum principle, there exists a maximal set $S_{\epsilon}$ in the family $\mathcal{F}_{\epsilon}$. Since $\bar{S}_{\epsilon} \in \mathcal{F}_{\epsilon}$, the set $S_{\epsilon}$ must be closed and therefore compact. Thus the time function must attain a minimum at some point $Z$ in the set $S_{\epsilon}$. This point $Z$ must be in $A$, since otherwise we could enlarge $S_{\epsilon}$ by adding to it a point in $A$ close to $Z$ and preceding it.

Now let $K$ be a subsequential limit (in Hausdorff convergence of sets) as $\epsilon \rightarrow 0$ of the $S_{\epsilon}$.

In addition to this maximum principle, we use two other facts about classical flows:

(i). If $M$ is a compact $C^{1,1}$ embedded hypsersurface at time $t$ (i.e., $M \subset$ $[t] \times N)$ then $M$ is the heat boundary of a classical flow $\mathcal{M}$. 
(ii). If $U$ is an open subset of spacetime and $U^{\prime}$ is the union of all classical flows with heat boundaries is $U$, then $U^{\prime}$ is also open.

(When the ambient space is euclidean, (ii) is trivial because a translate in spacetime of a classical flow is also a classical flow.)

\section{Weak Flows.}

Definition. Let $\Gamma$ be a closed subset of spacetime $\mathbf{R}^{+} \times N$. (Throughout this paper, $\mathbf{R}^{+}=[0,+\infty)$.) A closed set $\mathcal{M} \subset \mathbf{R}^{+} \times N$ is said to be a weak flow generated by $\Gamma$ provided

(i). $\mathcal{M}$ and $\Gamma$ coincide at time 0 .

(ii). If $\mathcal{M}^{\prime}$ is a classical flow with $\partial \mathcal{M}^{\prime}$ disjoint from $\mathcal{M}$ and with $\mathcal{M}^{\prime}$ disjoint from $\Gamma$, then $\mathcal{M}^{\prime}$ is disjoint from $\mathcal{M}$.

Let $\mathcal{M}$ be a classical flow and $\Gamma$ be its heat boundary. Then by the maximum principle lemma $3.1, \mathcal{M}$ is a weak flow generated by $\Gamma$.

One can make a slightly different definition of weak flow by requiring that the $\mathcal{M}^{\prime \prime}$ 's in (ii) be classical flows of closed hypersurfaces. It is not hard to show that the resulting definition is equivalent to the one we have given, so the reader can use whichever he or she prefers.

Let $\tau: \mathbf{R}^{+} \times N \rightarrow \mathbf{R}^{+} \times N$ be a translation forward in time: $\tau(t, x)=$ $(t+T, x)$ Note that $\mathcal{M}$ is a weak flow generated by $\Gamma$ if and only if $\tau(\mathcal{M})$ is a weak flow generated by $\tau(\Gamma)$.

Weak flows have a restarting property. Suppose $\mathcal{M}$ is a weak flow generated by $\Gamma$. Fix a $T>0$ and let

$$
\begin{aligned}
& \Gamma^{\prime}=\{(t, x) \in \Gamma: t \geq T\} \cup\{(t, x) \in \mathcal{M}: t=T\} \\
& \mathcal{M}^{\prime}=\{(t, x) \in \mathcal{M}: t \geq T\}
\end{aligned}
$$

Then $\mathcal{M}^{\prime}$ is a weak flow generated by $\Gamma^{\prime}$.

Also, weak flows can "pop" at any time. Let $\mathcal{M}$ be a weak flow generated by $\Gamma$. Fix a $T \geq 0$, and let

$$
\mathcal{M}^{\prime}=\Gamma \cup\{(t, x) \in \mathcal{M}: t \leq T\}
$$

Then $\mathcal{M}^{\prime}$ is another weak flow generated by $\Gamma$.

Theorem 4.1 (Avoidance Theorem). For $i=1,2$, suppose $\mathcal{M}_{i}$ is a weak flow generated by $\Gamma_{i}$. Suppose each initial portion $\left\{(t, x) \in \mathcal{M}_{1}: t \leq\right.$ 
$\tau$ \} of $\mathcal{M}_{1}$ is compact. Suppose also that at some time $T$,

$$
\operatorname{dist}_{T}\left(\mathcal{M}_{1}, \mathcal{M}_{2}\right)<\min _{i \neq j} \operatorname{dist}_{T}\left(\mathcal{M}_{i}, \Gamma_{j}\right)
$$

Then $\operatorname{dist}_{t}\left(\mathcal{M}_{1}, \mathcal{M}_{2}\right)$ is a non-increasing function of $t$ on some interval $[T, T+$ $\epsilon]$.

Proof for euclidean space. (Ilmanen [I1, §10] or [I3, §4].) Let $M$ be a compact $C^{1,1}$ surface contained in $\left\{(T, x) \in \mathbf{R}^{+} \times N\right\}$ such that $M$ separates $\mathcal{M}_{1}$ from $\mathcal{M}_{2}$ at time $T$ and such that

$$
\operatorname{dist}_{T}\left(\mathcal{M}_{1}, \mathcal{M}_{2}\right)=\operatorname{dist}_{T}\left(\mathcal{M}_{1}, M\right)+\operatorname{dist}_{T}\left(M, \mathcal{M}_{2}\right)
$$

(Existence of such an $M$ is proved in $[\mathrm{I} 3, \S 4 \mathrm{~F}]$.) Let $\mathcal{M}^{\prime}$ be a classical flow generated by $M$. Then for a short time interval $T<t<T+\epsilon$,

$$
\operatorname{dist}_{t}\left(\mathcal{M}, \mathcal{M}_{i}\right) \leq \operatorname{dist}_{T}\left(\mathcal{M}, \mathcal{M}_{i}\right)
$$

(Otherwise, by translating $\mathcal{M}$ spatially we would get a classical flow $\mathcal{M}^{\prime}$ that was disjoint from $\mathcal{M}_{i}$ at time $T$ and then intersecting it at a slightly later time, contrary to the definition of weak flow.) Thus

$$
\begin{aligned}
\operatorname{dist}_{t}\left(\mathcal{M}_{1}, \mathcal{M}_{2}\right) & \geq \operatorname{dist}_{t}\left(\mathcal{M}_{1}, \mathcal{M}\right)+\operatorname{dist}_{t}\left(\mathcal{M}, \mathcal{M}_{2}\right) \\
& \geq \operatorname{dist}_{T}\left(\mathcal{M}_{1}, \mathcal{M}\right)+\operatorname{dist}_{T}\left(\mathcal{M}, \mathcal{M}_{2}\right) \\
& \geq \operatorname{dist}_{T}\left(\mathcal{M}_{1}, \mathcal{M}_{2}\right)
\end{aligned}
$$

See $\S 7$ for a proof of the avoidance theorem for arbitrary ambient manifolds.

Ilmanen [I2] gives various interesting examples of compact surfaces that become non-compact in finite time under the mean curvature flow. Fortunately he also gives a natural condition that precludes this:

Theorem 4.2. Suppose $N$ is complete with ricci curvature bounded below. Let $\mathcal{M}$ be a weak flow generated by $\Gamma \subset \mathbf{R}^{+} \times N$. If $\Gamma \cap\{(t, x): t \leq \tau\}$ is compact, then so is $\mathcal{M} \cap\{(t, x): t \leq \tau\}$. 


\section{Level Set Flow.}

Definition. Let $\Gamma$ be a closed subset of spacetime $\mathbf{R}^{+} \times N$. We define a family of subsets of $W_{k}=W_{k}(\Gamma)$ of spacetime inductively as follows.

$$
W_{0}=\{(0, x):(0, x) \notin \Gamma\}
$$

Given $W_{k}$, we let $W_{k+1}$ be the union of all classical flows $\mathcal{M}^{\prime}$ such that $\mathcal{M}^{\prime}$ is disjoint from $\Gamma$ and such that the heat boundary of $\mathcal{M}^{\prime}$ is contained in $W_{k}$.

Finally we let $W=\cup_{k=0}^{\infty} W_{k}$ and

$$
\mathcal{M}(\Gamma)=\mathbf{R}^{+} \times N \backslash W
$$

This set $\mathcal{M}(\Gamma)$ is called the level set flow or biggest flow generated by $\Gamma$.

Note that each $W_{k}$ (with $k>0$ ) is open, and that $W_{0} \subset W_{1} \subset W_{2} \subset \ldots$

Proposition 5.1. Let $\mathcal{M}$ be the level set flow generated by $\Gamma$. Then $\mathcal{M}$ is a weak flow, and if $\tilde{\mathcal{M}}$ is any other weak flow generatēd by $\Gamma$, then $\tilde{\mathcal{M}} \subset \mathcal{M}$.

Proof. Note by induction that every $W_{k}$ is contained in the complement of $\tilde{\mathcal{M}}$. Thus $W \subset \tilde{\mathcal{M}}^{c}$, so $\tilde{\mathcal{M}} \subset \mathcal{M}$. This proves the second assertion.

Now suppose $\mathcal{M}^{\prime}$ is a classical flow with $\mathcal{M}^{\prime}$ disjoint from $\Gamma$ and $\partial \mathcal{M}^{\prime}$ contained $W$ (the complement of $\mathcal{M}$.) Then by compactness, $\partial \mathcal{M}^{\prime}$ is contained in one of the $W_{k}$. Thus by definition of $W_{k+1}, \mathcal{M}^{\prime}$ is contained in $W_{k+1}$ and therefore in $W$. This proves that $\mathcal{M}$ is a weak solution generated by $\Gamma$.

Theorem 5.2. Let $\mathcal{M}$ be the level set flow generated by $\Gamma$. Then

(i). For each $X \in \mathcal{M}^{c}=W$, there is a time-like path in $W$ joining $X$ to a point $Y=(0, y)$ at time 0 .

(ii). If $X$ and $Y$ are in different connected components of $W_{0}$, then they are in different connected components of $W$.

Proof. To prove (i), let $X \in W$. Then $X \in W_{k}$ for some $k$. Let us prove the result by induction on $k$. If $k=0$, we are done. If $k>0$, then by definition there is a classical flow $\mathcal{M}^{\prime}$ with $X \in \mathcal{M}^{\prime}$ and $\partial \mathcal{M}^{\prime} \in W_{k-1}$. Trivially $X$ is connected (back through $\mathcal{M}^{\prime}$ ) to a point $Y$ in the initial part of $\mathcal{M}^{\prime}$. Since $\mathcal{M}^{\prime} \subset W_{k-1}$, by induction we can connect $Y$ back to a point in $W_{0}$. 
To prove (ii), suppose that two points $X, Y \in W_{0}$ cannot be connected by a path in $W_{0}$. Then $W_{0}$ can be written as the union of two disjoint open sets $W_{0}^{X}$ and $W_{0}^{Y}$ containing $X$ and $Y$, respectively. Let $W_{k}^{X}$ be the union of all classical flows $\mathcal{M}^{\prime}$ that are disjoint from $\Gamma$ and that have heat boundary in $W_{k-1}^{X}$, and similarly for $W_{k-1}^{Y}$.

If there were a point $Z$ in both $W_{j}^{X}$ and $W_{k}^{Y}$, then $Z$ would belong to a classical flow $\mathcal{M}^{\prime}$ disjoint from $\Gamma$ and with heat boundary in $W_{j-1}^{X}$ and to a classical flow $\mathcal{M}^{\prime \prime}$ disjoint from $\Gamma$ and with heat boundary in $W_{k-1}^{Y}$. By the maximum principle lemma 3.1, it follows that either $W_{j-1}^{X}$ intersects $W_{k}^{Y}$ or $W_{j}^{X}$ intersects $W_{k-1}^{Y}$. Repeating the process, we eventually find that $W_{i}^{X}$ intersects $W_{0}^{Y}$ or that $W_{0}^{X}$ intersects $W_{i}^{Y}$, both of which are clearly impossible. This shows that $W_{k}^{X}$ is dijoint from $W_{k}^{Y}$. Thus $\cup_{k} W_{k}^{X}$ is disjoint from $\cup_{k} W_{k}^{Y}$. But the union of these two open sets is all of $W$, so $X$ and $Y$ belong to different components of $W$. This proves (ii).

For the next theorem we need the following lemma about the sets $W_{k}$ used in the definition of the level set flow generated by $\Gamma$ :

Lemma 5.3. Let $\mathcal{M}^{\prime}$ be a classical flow that is disjoint from $\Gamma$ and whose heat boundary is contained in $W_{k}$. Then each connected component of $\mathcal{M}^{\prime} \cap W_{k}$ contains points in $\partial \mathcal{M}^{\prime}$.

Proof. Let $V$ be a connected component of $\mathcal{M}^{\prime} \cap W_{k}$. Let $j$ be the smallest number such that $V$ intersects $W_{j}$, and let $X$ be a point in $V \cap W_{j}$. Of course $j \leq k$. If $j=0$, we are done. If not, then by definition of $W_{j}$, there is a classical flow $\mathcal{M}^{*}$ such that $X \in \mathcal{M}^{*}, \mathcal{M}^{*}$ is disjoint from $\Gamma$, and $\partial \mathcal{M}^{*} \subset W_{j-1}$. By the maximum principle lemma 3.1, there is a connected subset $K$ of $\mathcal{M}^{\prime} \cap \mathcal{M}^{*}$ such that $K$ contains both $X$ and a point $Y$ in $\partial \mathcal{M}^{\prime} \cup \partial \mathcal{M}^{*}$. Since $\mathcal{M}^{*} \subset W_{j} \subset W_{k}, K \subset V$. Since $\partial \mathcal{M}^{*} \subset W_{j-1}$, $Y \notin \partial \mathcal{M}^{*}$ (by the choice of $j$ ), so $Y \in \partial \mathcal{M}^{\prime}$.)

Theorem 5.4. Let $\mathcal{M}$ be a level set flow, and let $W[0, T]$ denote the portion from $t=0$ to $t=T$ of the complement of $\mathcal{M}$ :

$$
W[0, T]=\{(t, x) \notin \mathcal{M}: 0 \leq t \leq T\}
$$

Then every closed loop in $W[0, T]$ is homotopic (in this set) to a closed loop in $W[0]$. 
Proof. Let $W_{k}$ be as in the definition of the level set flow $\mathcal{M}$ generated by $\Gamma$. Let $W_{k}[0, T]$ be the portion of $W_{k}$ from time $t=0$ to $t=T$. By the basic theory of covering spaces, there is a covering space

$$
\Pi: \tilde{W}[0, T] \rightarrow W[0, T]
$$

such that a loop $\gamma$ in $W[0, T]$ lifts to $\tilde{W}[0, T]$ if and only if it is homotopic to a loop in $W[0]$. This immediately implies that there is a lift

$$
\tilde{\iota}_{0}: W[0] \rightarrow \tilde{W}[0, T]
$$

of the inclusion map $\iota_{0}: W[0] \rightarrow W[0, T]$. (Recall that $\tilde{f}$ is a lift of $f$ if $f=\Pi \circ \tilde{f}$.)

We now construct inductively for each $k$ a lift

$$
\tilde{\iota}_{k}: W_{k}[0, T] \rightarrow \tilde{W}[0, T]
$$

of the inclusion map $\iota_{k}: W_{k}[0, T] \rightarrow W[0, T]$ such that $\iota_{k}$ agrees with $\iota_{k-1}$ on $W_{k-1}[0, T]$.

Thus suppose we are given a lift $\tilde{\iota}_{k-1}$ of $\iota_{k-1}$, and let $\mathcal{M}^{\prime}$ be a classical flow disjoint from $\Gamma$ with heat boundary in $W_{k-1}$. Then there is a unique lift

$$
\phi: \mathcal{M}^{\prime} \rightarrow \tilde{W}[0, T]
$$

such that

$$
\phi(X)=\tilde{\iota}_{k-1}(X) \text { for } X \in \partial \mathcal{M}^{\prime}
$$

(The lift is constructed as follows. Choose some parametrization $F:[a, b] \times$ $M \rightarrow \mathcal{M}^{\prime}$ as in the definition of classical flows (§3). For each $p \in M$, lift the path $t \mapsto F(t, p)$ to $\tilde{W}[0, T]$, starting at $t=a$ at $\tilde{\iota}_{k-1}(F(a, p)$.)

Claim 1. $\phi(X)=\tilde{\iota}_{k-1}(X)$ for every $X \in \mathcal{M}^{\prime} \cap W_{k-1}[0, T]$.

Proof of claim 1. Let $V$ be a connected component of $\mathcal{M}^{\prime} \cap W_{k-1}$. Then $\phi$ and $\tilde{\iota}_{k-1}$ give two lifts of $V$ into $\tilde{U}$. By lemma 5.3, $V$ must contain scme point $Y$ of $\partial \mathcal{M}^{\prime}$. By $\left({ }^{*}\right)$, these two lifts agree at $Y$. Thus they must agree throughout $V$. This proves claim 1 .

Claim 2. Let $\mathcal{M}_{1}$ and $\mathcal{M}_{2}$ be two classical flows disjoint from $\Gamma$ and with heat boundaries in $W_{k-1}$, and let $\phi_{1}$ and $\phi_{2}$ be the corresponding lifts. If $X \in \mathcal{M}_{1} \cap \mathcal{M}_{2}$, then $\phi_{1}(X)=\phi_{2}(X)$. 
Proof of claim 2. By the maximum principle lemma 3.1, there is a connected subset $K$ of $\mathcal{M}_{1} \cap \mathcal{M}_{2}$ containing $X$ and some point $Y \in \partial \mathcal{M}_{1} \cup \partial \mathcal{M}_{2}$. By claim $1, \phi_{1}(Y)=\tilde{\iota}_{k-1}(X)=\phi_{2}(Y)$. Since $\phi_{1}$ and $\phi_{2}$ give two lifts of $K$ that agree at $Y$, they must also agree at $X$. This proves claim 2 .

Now we extend $\tilde{\iota}_{k-1}: W_{k-1}[0, T] \rightarrow \tilde{W}[0, T]$ to $\tilde{\iota}_{k}: W_{k}[0, T] \rightarrow \tilde{W}[0, T]$ as follows. Given $X \in W_{k}$, there exists a classical flow $\mathcal{M}^{\prime}$ with $X \in \mathcal{M}^{\prime}$ and $\partial \mathcal{M}^{\prime} \subset W_{k-1}$. Let $\phi: \mathcal{M}^{\prime} \rightarrow \tilde{U}$ be the lift described above. Then we let $\tilde{\iota}_{k}(X)=\phi(X)$; note by claim 2 this does not depend on the choice of $\mathcal{M}^{\prime}$.

Putting the $\tilde{\iota}_{k}$ 's together, we get a lift

$$
\tilde{\iota}: W[0, T] \rightarrow \tilde{W}[0, T]
$$

This means that $\tilde{W}[0, T]=W[0, T]$, which implies the theorem.

\section{More on Weak Flows.}

Throughout this section we assume that the ambient space $N$ is a complete $(n+1)$-dimensional manifold with ricci curvature bounded below.

Theorem 6.1. Let $\mathcal{M}$ be a weak flow generated by a set $M_{0}$ at time 0 . Let $W$ be the complement of $\mathcal{M}$, and let

$$
\begin{aligned}
W[T] & =\{(t, x) \in W: t=T\} \\
W[0, T] & =\{(t, x) \in W: 0 \leq t \leq T\}
\end{aligned}
$$

If $C$ is a polyhedral $(n-1)$ cycle in $W[T]$ that is homologically trivial in $W[0, T]$, then it is homologically trivial in $W[T]$.

In other words, the inclusion of $W[T]$ into $W[0, T]$ induces a monomorphism on $\mathbf{H}_{n-1}$.

Proof. Let $\Gamma$ be the support of an polyhedral $n$-chain $P$ in $W[0, T]$ with $\partial P=C$. Note we may choose $\Gamma$ so that its $t=0$ slice is empty. Let $\mathcal{M}^{\prime}$ be the level set flow generated by $\Gamma$. By theorem 4.2, the portion of $\mathcal{M}^{\prime}$ up to any time $t$ is compact.

Let $\epsilon=\min _{0 \leq t \leq T} \operatorname{dist}_{t}(\Gamma, \mathcal{M})$. Note that $\epsilon>0$. By the avoidance theorem 4.1,

$$
\operatorname{dist}_{t}\left(\mathcal{M}^{\prime}, \mathcal{M}\right) \geq \epsilon \quad(0 \leq t \leq T)
$$


Now fatten $\mathcal{M}^{\prime}[T]$ a little to get a set $K$ in $[T] \times N$ that is disjoint from $\mathcal{M}[T]$ and that has smooth boundary.

Let

$$
\begin{aligned}
& \pi:[0, T] \times N \rightarrow[T] \times N \\
& \pi(t, x)=(T, x)
\end{aligned}
$$

Let $P^{\prime}=\pi_{\#} P$. Then $\partial P^{\prime}=\partial \pi_{\#} P=\pi_{\#} \partial P=C$.

Suppose $\gamma$ is a loop in $([T] \times N) \backslash K$. Then $\gamma$ is in the complement; of $\mathcal{M}^{\prime}$, so it is homotopic in this complement to a loop at time $t=0$. Note this implies that the algebraic intersection number of $\gamma$ with $P^{\prime}$ is 0 .

Now assign multiplicities to each region in the complement (in $[T] \times N)$ of $K \cup P^{\prime}$ in such that the multiplicity changes by $\alpha$ whenever we cross a face of $P^{\prime}$ with multiplicity $\alpha$. Note this is possible to do consistently because any loop in the complement of $K$ has (algebraic) intersection number 0 with $P^{\prime}$. The result is an $(n+1)$-chain $Q$ whose boundary is the portion $P^{\prime} \backslash K$ of $P^{\prime}$ outside of $K$ together with a chain $Q$ in $K$.

Thus if we modify $P^{\prime}$ by replacing $P^{\prime} \backslash K$ with $Q$, we get an $n$-chain in $K$ (and therefore disjoint from $\mathcal{M}$ ) whose boundary is the same as the boundary of $P^{\prime}$, namely $C$.

Theorem 6.2. Let $\mathcal{M}$ be a weak flow generated by a set $M_{0}$ at time 0 . Then (in the notation of theorem 6.1), if a polyhedral $n$-cycle $C$ in $W[T]$ bounds a $(n+1)$-chain in $W[0, T]$, then it bounds an $(n+1)$-chain in $W[T]$.

In other words, the inclusion of $W[T]$ into $W[0, T]$ induces a monomorphism on $\mathbf{H}_{n}$.

Proof. Let $\Gamma$ be the support of an polyhedral $(n+1)$-chain $P$ in $W[0, T]$ with $\partial P=C$. Note we may choose $\Gamma$ so that its $t=0$ slice is empty. Let $\mathcal{M}^{\prime}$ be the level set flow generated by $\Gamma$. By theorem 4.2, the portion of $\mathcal{M}^{\prime}$ up to time $t$ is compact.

Let $\epsilon=\min _{0 \leq t \leq T} \operatorname{dist}_{t}(\Gamma, \mathcal{M})$. Note that $\epsilon>0$. By the avoidance theorem 4.1,

$$
\operatorname{dist}_{t}\left(\mathcal{M}^{\prime}, \mathcal{M}\right) \geq \epsilon \quad(0 \leq t \leq T)
$$

Let $\pi$ be as in the proof of theorem 6.1 , and let $P^{\prime}=\pi_{\#} P$. Then $\partial P^{\prime}=\partial \pi_{\#} P=\pi_{\#} \partial P=C$.

We claim that the support of $P^{\prime}$ is contained in $\mathcal{M}^{\prime}$, and is therefore disjoint from $\mathcal{M}$. For let $X=(T, x)$ be in the support of $P^{\prime}$ but not in the support of $C$. Then the path $t \mapsto(t, x)(0 \leq t \leq T)$ intersects $P$ a nonzero 
number $\alpha$ of times (counting multiplicities). Thus any path joining $X$ to a point $\left(0, x^{\prime}\right)$ intersects $P \alpha$ times. Hence $X$ cannot be connected to any point with $t=0$ by a path disjoint from $\Gamma$. Thus by theorem $5.2(\mathrm{i}), X \in \mathcal{M}^{\prime}$.

\section{Riemannian Manifolds.}

All the theorems in the preceding sections have been stated for general ambient riemannian manifolds. However, the proof of the avoidance theorem was valid only in euclidean spaces (and flat tori). In this section we give a proof for other ambient manifolds.

Theorem 7.1 (Avoidance Theorem). Suppose $N$ is complete with Ricci curvature bounded below. For $i=1,2$, suppose $\mathcal{M}_{i}$ are weak flows generated by $\Gamma_{i}$. Suppose:

(i). the initial portion $\left\{(t, x) \in \mathcal{M}_{1}: t \leq T\right\}$ of $\mathcal{M}_{1}$ is compact,

(ii). $\mathcal{M}_{1}$ and $\mathcal{M}_{2}$ are disjoint at time $t=0$,

(iii). $\min _{i \neq j} \operatorname{dist}_{t}\left(\mathcal{M}_{i}, \Gamma_{j}\right)>\epsilon>0$ for $0 \leq t \leq T$.

Then $\mathcal{M}_{1}$ and $\mathcal{M}_{2}$ are disjoint up to time $T$.

Proof. Suppose not. Then there is a first time of contact, which without loss of generality we may suppose to be $T$. Note the contact set is a compact set $K$. Let $K^{\prime}$ be a compact set whose interior contains $K$. If we replace $\mathcal{M}_{2}$ by $\mathcal{M}_{2} \cap\left(\mathbf{R}^{+} \times K^{\prime}\right)$ and $\Gamma$ by

$$
\left(\Gamma \cap\left(\mathbf{R}^{+} \times K^{\prime}\right)\right) \cup\left(\mathcal{M}_{2} \cap\left(\mathbf{R}^{+} \times \partial K^{\prime}\right)\right)
$$

then the hypotheses are still satisfied (perhaps with a smaller $\epsilon$ ), except that now the portion of $\mathcal{M}_{2}$ up to and including time $t=T$ is compact.

Fix a $T^{\prime}<T$. Let

$\Gamma_{i}^{\prime}=\left\{X=(t, x): t=T^{\prime}, \operatorname{dist}_{t}\left(X, \Gamma_{i}\right) \leq \epsilon / 3\right\} \cup\left\{X=(t, x): t=T^{\prime}, X \in \mathcal{M}_{i}\right\}$

Let $\mathcal{M}_{i}^{\prime}$ be the biggest flow generated by $\Gamma_{i}^{\prime}$. Note that if we choose $T^{\prime}$ sufficiently close to $T$, then $\Gamma_{i}$ will be in the interior of $\mathcal{M}_{i}^{\prime}$ for all times $t \in\left[T^{\prime}, T\right]$. 
It follows immediately that $\mathcal{M}_{i} \subset \mathcal{M}_{i}^{\prime}$ in the time interval $t \in\left[T^{\prime}, T\right]$. But during this time interval the $\mathcal{M}_{i}^{\prime}$ are flowing according to the level set flow without boundary as considered in [I2]. Thus according to the results of that paper (in particular theorems 6.3 and 6.4), the $\mathcal{M}_{i}^{\prime}$ are disjoint. Hence the $\mathcal{M}_{i}$ are disjoint (up to time $T$ ), a contradiction.

Remark. It would be nice to prove 7.1 in a direct geometrical way, without using viscosity solutions.

\section{Other Curvature Flows.}

The results of this paper hold for some flows other than the mean curvature flow. Suppose we have an evolution equation for hypersurfaces in $\mathbf{R}^{i+1}$ such that:

(i). the equation is translation invariant,

(ii). the equation does not depend on orientation of the surface,

(iii). classical solutions satisfy the strong maximum principle,

(iv). for any compact $C^{1,1}$ embedded hypersurface in $\mathbf{R}^{n+1}$, there is a classical solution for some (possibly short) time interval.

Then we can define corresponding generalized solutions (weak flows and level set flows), and all the results of this paper continue to hold (with exactly the same proofs).

Examples of such evolution equations can be obtained by letting a surface flow by the first variation vectorfield corresponding to any constantcoefficient even elliptic integrand.

The results would also hold for an evolution equation in a compact ambient manifold $N$ satisfying (ii),(iii), and (iv), provided one could prove the strong avoidance property 7.1.

For non-compact ambient manifolds, one would need to prove the avoidance property and one would also need to replace the assumption about ricci curvature by some condition (perhaps something like Morrey's homogeneous regularity) that would let one prove the analogue of 4.2. The other proofs would then work exactly as before. 


\section{References.}

[AAG] S. Altschuler, S. Angenent, and Y. Giga, Mean curvature flow through singularities for surfaces of rotation, Hokkaido Univ. Preprint Series in Math. 130(1991).

[B] K. Brakke, The motion of a surface by its mean curvature, Princeton U. Press 1978.

[CGG] Y. G. Chen, Y. Giga, and S. Goto, Uniqueness and existence of viscosity solutions of generalized mean curvature flow equations, J. Diff. Geom. 33(1991), 749-786.

[ES] L. C. Evans and J. Spruck, Motion of level sets by mean curvature I, J. Diff. Geom. 33(1991), 635-681.

[H] Asymptotic behavior for singularities of the mean curvature flow, J. Diff. Geom. 31(1990), 285-299.

[I1] T. Ilmanen, Elliptic regularization and partial regularity for motion by mean curvature, Memoirs of the Amer. Math. Soc. 108(520)(1994).

[I2] Generalized flow of sets by mean curvature on a manifold, Indiana Univ. Math. J. 41(1992), 671-705.

[13] The level-set flow on a manifold, Proc. 1990 Summer Institute Diff. Geom.

[OS] S. Osher and J. Sethian, Fronts propagating with curvature-dependent speed: algorithms based on Hamilton-Jacobi formulations, J. Comput. Phys. 79(1988), 12-49.

[S] M. Soner, Motion of a set by the curvature of its boundary, J. Diff. Eq. 101(1993), 313-372.

[W1] B. White, Partial regularity of mean-convex hypersurfaces flowing by mean curvature, Int. Math. Research Notices 4, 1994, 185-192.

[W2] On the topological type of minimal submanifolds, Topology 31(2)(1992), 445-448.

STANFORD UNIVERSITY

STANFORD, CA 94305

E-MAIL: WHITE@CAUCHY.STANFORD.EDU

RECEIVEd OCTOBER 3RD, 1994. 